\title{
¿Genesis of Upper-Tropospheric Anticyclones over the Asian-Western Pacific Sector from Tropical-Extratropical Interaction Perspective
}

\author{
Hiroaki Ueda, ${ }^{\mathrm{a}}$ Masaya Kuramochi,${ }^{\mathrm{b}}$ Koutarou TAKaya, ${ }^{\mathrm{c}}$ YUhei TAKaya, ${ }^{\mathrm{d}}$ SaKi Asano, ${ }^{\mathrm{a}}$ and \\ SHUHEI MAEDA ${ }^{\mathrm{e}}$ \\ ${ }^{\text {a }}$ Faculty of Life and Environmental Sciences, University of Tsukuba, Tsukuba, Japan \\ ${ }^{\mathrm{b}}$ Graduate School of Life and Environmental Sciences, University of Tsukuba, Tsukuba, Japan \\ ${ }^{\mathrm{c}}$ Department of Physics, Faculty of Science, Kyoto Sangyo University, Kyoto, Japan \\ ${ }^{\mathrm{d}}$ Meteorological Research Institute, Tsukuba, Japan \\ e Aerological Observatory, Tsukuba, Japan
}

(Manuscript received 8 January 2021, in final form 8 November 2021)

\begin{abstract}
Upper-tropospheric anticyclones (UTACs) emerge throughout the seasons with changing location and intensity. Here, the formation mechanisms of these UTACs, especially in the Asian-Australian-western Pacific sector, were investigated based on the diagnosis of the vorticity equation as well as the contribution of the planetary waves. During June-July-August (JJA), a vigorous UTAC corresponding to the South Asian high (SAH) forms over South Asia, to the south of the Tibetan Plateau, where intense heating associated with the Asian summer monsoon rainfall and the resultant baroclinic Rossby response are the important physical processes. Meanwhile, the produced anticyclonic vorticity is farther transported by the interhemispheric divergent wind toward the Southern Hemisphere (SH), creating the SH UTAC centered over the Maritime Continent. During December-January-February (DJF), two zonally elongated UTACs reside on each side of the equator $\left(\sim 10^{\circ}\right.$ poleward), mainly over the Maritime Continent-western Pacific sector. Upon a closer look at the NH winter, we observed that the northern parts of UTAC cannot be explained by this vorticity balance alone. Diagnosis of the wave activity flux indicated that planetary waves emanating from the cold Eurasian continent converges around the northern parts of the UTAC with its peak in the NH winter, which weakens the subtropical jet, thus generating UTAC. Configuration of the SH summer (DJF) UTAC bears resemblance with that of SAH. These results suggest that the creation of anticyclonic vorticity and its interhemispheric transportation as well as the propagation of planetary wave are the selectively important agents for the genesis of seasonally varying UTACs.
\end{abstract}

SIGNIFICANCE STATEMENTS: Recent studies have provided evidence that the South Asian high (formerly Tibetan high) is not a purely thermally driven system only maintained over the elevated Tibetan Plateau. This study aims to understand the physical processes responsible for the genesis of the upper-tropospheric anticyclone, especially in the Asian-Australian-western Pacific sector, throughout the season. During summer in the Northern Hemisphere, deep heating caused by South Asian monsoon rainfall plays a crucial role in the genesis of the South Asian high. The wintertime anticyclone emerging over the subtropical western North Pacific is caused via remote influences anchored with tropical convection and the cold Eurasian continent in which atmospheric teleconnections are important. These findings provide new avenues for research on tropical-extratropical interactions with respect to the formation and variability of important climate phenomena.

KEYWORDS: Asia; Pacific Ocean; Anticyclones; Atmospheric circulation; Rossby waves; Teleconnections; Climatology

\section{Introduction}

Since the recognition of the vigorous upper-tropospheric anticyclone (UTAC) emerging over South Asia to the south of the Tibetan Plateau in the northern summer (Flohn 1957; Krishnamurti 1971a,b), its formation process has received renewed attention. The identification of the high pressure system, which was formerly known as the Tibetan high but now more frequently referred to as the South Asian high (SAH), is considered a major advancement in Asian monsoon research. The orographic effect, namely, a combination of

¿Denotes content that is immediately available upon publication as open access. ac.jp sensible heat supply from the elevated plateau (Murakami 1987; Yanai et al. 1992) and latent heating along the Himalayas (Ueda et al. 2003; Wu and Liu 2003), has been thought to drive the Asian summer monsoon. Observational studies suggest that the SAH is not a purely thermally driven system caused by heating of the plateau, because its center is not only formed over the plateau but, rather, shifts southwestward over the Indian subcontinent. In addition to this, the upper anticyclones are also located over the western tropical Pacific to the east of the Philippines during the northern winter (Krishnamurti 1985), which does not accompany neighboring diabatic heating (Yanai and Tomita 1998). Several modeling studies with no mountains have shown that the deep condensation heating over South Asia is an essential process for the generation of the SAH through the dynamic atmospheric response to the heating (Hoskins and Rodwell 1995; Ose 1998; Abe et al. 2003; Boos and Kuang 2013). 
(a) PSI 200 , Omega 500 (JJA) + Convection center (months)

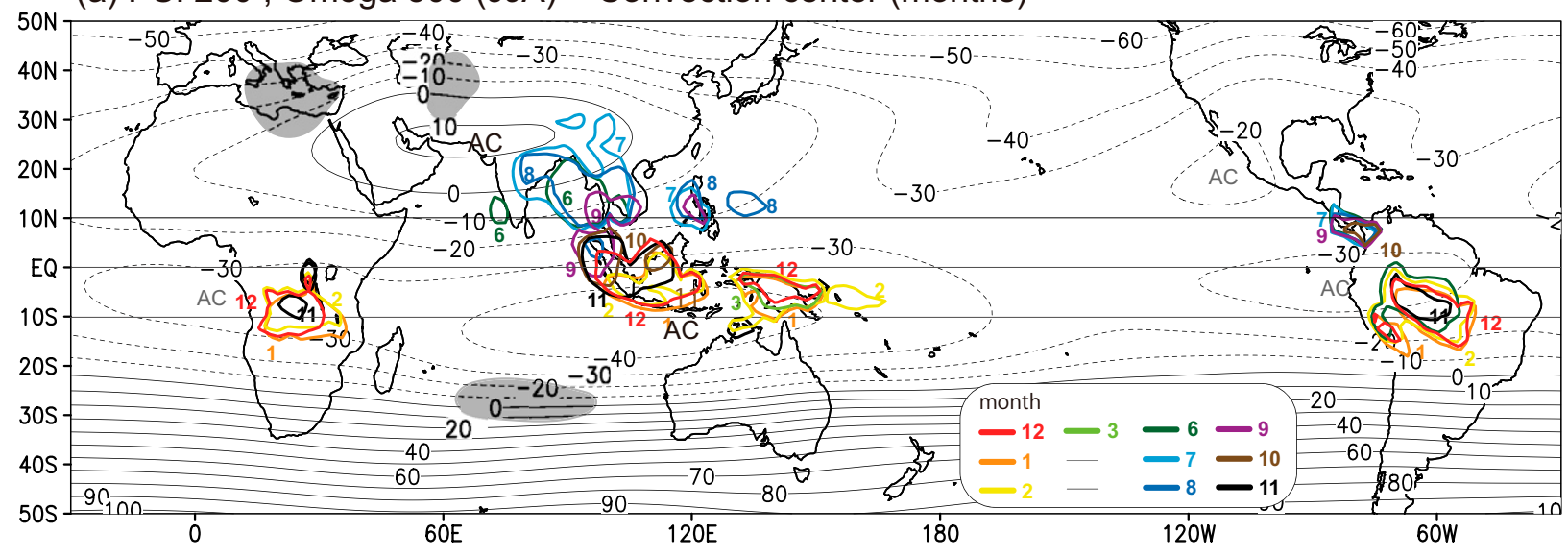

(b) PSI 200 , Omega 500 (DJF) + UTAC center (months)

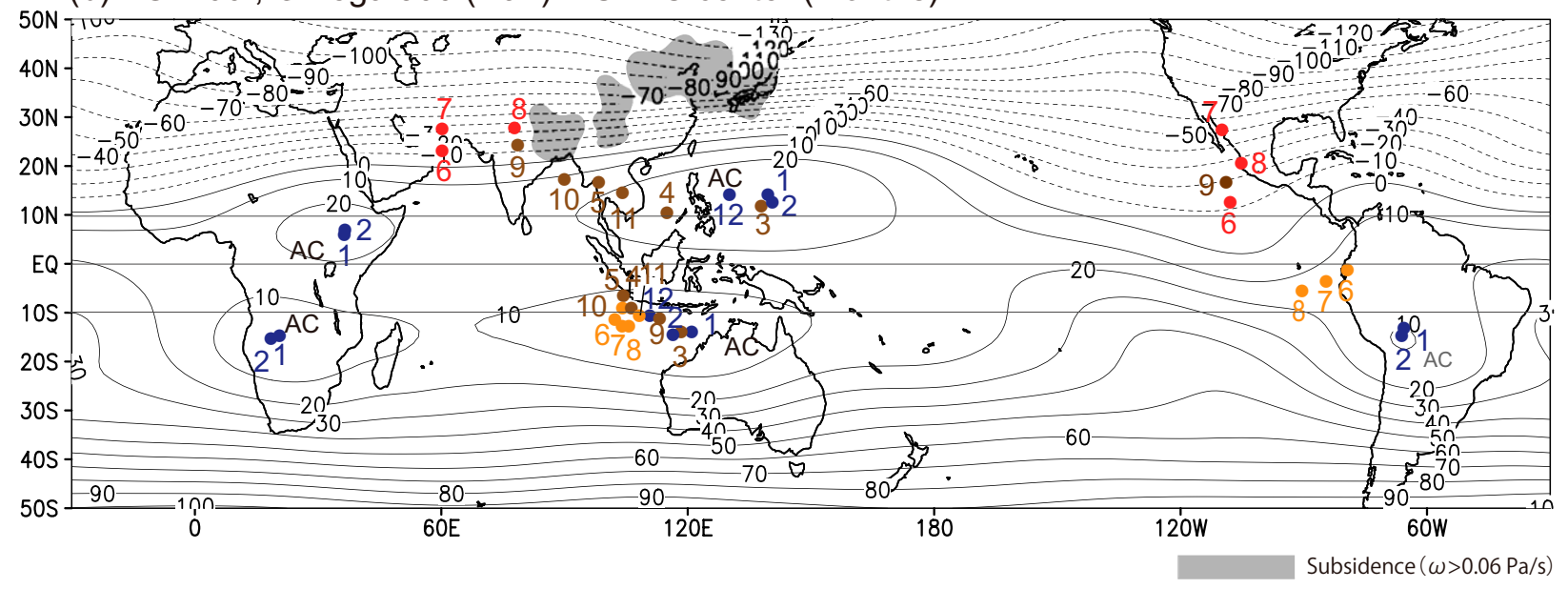

FIG. 1. Mean 200-hPa streamfunction for the (a) northern summer (June-August) and (b) southern summer (December-February). Regions of strong subsidence at $500 \mathrm{hPa}$ (vertical $p$ velocity greater than $6.0 \times 10^{-2} \mathrm{~Pa} \mathrm{~s}^{-1}$ ) are indicated by gray shading. Negative contours, indicated by dashed lines, show the streamfunction field. Colored contours are the seasonal migration of enhanced convection defined by monthly outgoing longwave radiation (OLR) values less than $200 \mathrm{~W} \mathrm{~m}^{-2}$ in (a) and dots are the subjectively specified anticyclone (AC) center in (b). The colors of the numbers near the dots in (b) denote the month.

To identify the characteristics of the UTAC, we present, in Fig. 1, the seasonal mean [June-July-August (JJA) and December-January-February (DJF)] streamfunction at $200 \mathrm{hPa}$ together with the annual variation in the position of the maximum center of the UTAC and the heating field. The extent of diabatic heat source is determined from the areas with outgoing longwave radiation (OLR) $<200 \mathrm{~W} \mathrm{~m}^{-2}$, which is denoted by colored contours as a function of the seasons. The most prominent feature in the northern summer streamfunction (Fig. 1a) is the presence of a massive anticyclone over the northwestern Indian subcontinent, recognized as the $\mathrm{SAH}$, between June and September (see also the dots in Fig. 1b). Meanwhile, UTACs are also recognizable around the American continent. In the $\mathrm{SH}$, an extensive UTAC centered over Asian-Australian-western Pacific extends westward to South Africa and farther beyond.

During JJA, the heating maxima are found to the southeast of the SAH (Figs. 1a, 2a). The configuration of the circulation and heating field is presumably a consequence of the atmospheric response to the diabatic heating from the Bay of Bengal to the western North Pacific (Ose 1998; Wu et al. 2015). As the season advances, the summer heat source over South Asia, including the southeastern foothills of the Himalaya, moves to the western tropical Pacific across the equator through the Maritime Continent "land bridge," reaching to the east of New Guinea during late winter (Lau and Chan 1983). Thereafter, the heating maxima returns to migrate northwestward along the same "land bridge" toward the northern summer.

During DJF (Fig. 1b), two zonally elongated UTACs exist on each side of the equator $\left(\sim 10^{\circ}\right.$ poleward) over the Maritime Continent sector as well as the African and American continent. In comparison with the JJA fields, the UTACs around the American continent become obscured and the SH UTAC over the Maritime Continent sector shrinks. During this period, a UTAC is clearly identified over the subtropical western North 
Pacific. Interestingly, the northern winter UTAC extending from the western Pacific to the South China Sea does not accompany diabatic heating in the neighboring regions, implying a mechanism completely different from those of the northern summer SAH. In the Southern Hemisphere (SH), the DJF UTAC from the south Indian Ocean to the northern part of Australia is likely consistent with the local heating maxima. The UTAC in the SH in the JJA field is located at approximately the same position as during DJF (Fig. 1b), without nearby diabatic heating. Thus, the pair of UTACs during the winter hemisphere and deep heating over the summer hemisphere is assumed to be associated with a form of interhemispheric teleconnection through Rossby wave propagation from a source located in the tropics (e.g., Gill 1980; Hoskins and Karoly 1981). Sardeshmukh and Hoskins (1988) demonstrated that the deep heating in the tropics and the associated upper-tropospheric divergence results in an anomalous vorticity in the subtropics, termed the Rossby wave source (RWS), that excites the Rossby wave trains connecting the tropical heat source and extratropical atmospheric circulation in both hemispheres (e.g., Trenberth et al. 1998). In this study, we first focus on the UTACs over the Asian-Australian sector then extend the discussion to other UTACs with respect to tropical-extratropical interactions.

Salient subsidence at $500 \mathrm{hPa}$ is found over Central Asia and the Mediterranean Sea during JJA (Fig. 1a), which is dynamically coherent with the westward propagation of Rossby waves generated by diabatic heating associated with Asian summer monsoon rainfall (Rodwell and Hoskins 1996; Wang et al. 2012). This pair of subsiding and rising motions is often referred to as the "monsoon-desert mechanism." It is interesting to note that other downward motions emerge over East Asia during DJF as well as in the south Indian Ocean during JJA, located westward of the UTAC. We issue a caveat regarding the applicability of "monsoon-desert mechanism" for East Asia because the area of enhanced subsidence corresponds to the advection of cold air originating from the Siberian high.

Given these similarities and differences between the winter and summer UTACs, the purpose of this study is to address how local and remote heating can create the UTACs with the particular focus on the JJA UTACs in the Asian-Australian sector as well as the DJF UTAC over the western North Pacific. Specifically, we consider the extent to which vorticity fields explain the presence of UTACs, the differences between the SAH and other UTACs, and the causes of these differences in the context of tropical-extratropical interactions. We first document the observed structures of UTAC in section 3. This is followed by a diagnosis of vorticity fields by means of the barotropic vorticity equation in pressure coordinates in section 4; we also examine the relationships between planetary waves and UTACs with respect to wave-mean flow interactions. Finally, we summarize our findings along with a discussion of some of the key issues that call for further investigation.

\section{Data and method}

The main datasets used in this study were from the Japanese 55-year Reanalysis Project (JRA-55), with a spatial resolution of $1.25^{\circ} \times 1.25^{\circ}$ over 37 vertical levels from 1000 to $1 \mathrm{hPa}$, and covering a 41-yr period from 1976 to 2016 . A detailed description of the data assimilation system that produced this dataset is given by Kobayashi et al. (2015). Additionally, the global monthly mean OLR flux data edited by the National Oceanic and Atmospheric Administration (Liebmann and Smith 1996), were also used in this study as a proxy for convective activity.

Diabatic heating $Q_{1}$, attributed primarily to the precipitation, was obtained using the atmospheric thermodynamic equation from the 6-h sequence of the JRA-55:

$$
\frac{\partial T}{\partial t}=-\mathbf{v} \cdot \nabla T+\omega\left(\frac{R T}{c_{p} p}-\frac{\partial T}{\partial p}\right)+\frac{Q_{1}}{c_{p}}
$$

where $T$ is the temperature, $\mathbf{v}$ is the horizontal wind, $\omega$ is the vertical $p$ velocity, and $c_{p}$ is the specific heat for dry air. As shown by Yanai et al. (1973), by vertically integrating Eq. (1) from $100 \mathrm{hPa}$ to the surface pressure $P_{s}$, we obtain the total column heating $\left(\mathrm{W} \mathrm{m}^{-2}\right)$ of the atmosphere $\left\langle Q_{1}\right\rangle$ contributed by radiation, precipitation, and surface sensible heat.

Motivated by previous studies suggesting the importance of vorticity advection by the divergent flow in producing the effective Rossby wave source (RWS), we examined vorticity fields by using the barotropic vorticity equation in pressure coordinates as in Sardeshmukh and Hoskins (1988):

$\left(\frac{\partial}{\partial t}+\mathbf{v} \cdot \nabla\right)(\zeta+f)=-(\zeta+f) D+\mathbf{k} \cdot\left(\frac{\partial \mathbf{v}}{\partial p} \times \nabla \omega\right)-\omega \frac{\partial \zeta}{\partial p}+F_{r}$,

where $\zeta$ is relative vorticity, $f$ is planetary vorticity, $\mathbf{v}$ is horizontal wind, $\omega$ is vertical $p$ velocity, $D$ is horizontal divergence equivalent to $\nabla \cdot v_{\chi}$, and $F$ is a frictional term. In the upper troposphere, the vertical advection, $\omega(\partial \zeta / \partial p)$, and tilting term $\mathbf{k} \cdot(\partial \mathbf{v} / \partial p \times \nabla \omega)$ can be neglected because the vertical velocity is relatively small (Sardeshmukh and Hoskins 1985). Decomposing the velocity field into divergent $v_{\chi}$ and rotational $v_{\psi}$ parts, and putting all the rotational terms on the lefthand side gives

$$
\frac{\partial \zeta}{\partial t}+v_{\psi} \cdot \nabla(\zeta+f)=-v_{\chi} \cdot \nabla(\zeta+f)-(\zeta+f) \nabla \cdot v_{\chi}+F_{r}
$$

The left-hand side of Eq. (3) is indicative of Rossby wave propagation. The first term on the right side denotes the advection of the vorticity by the divergent flow. The second term on the right-hand side represents the generation of vorticity by divergence, usually referred to as vorticity stretching. The two terms on the right-hand side are jointly called as the RWS, which can be grouped into as one:

$$
\mathrm{RWS}=-\nabla \cdot\left[v_{\chi}(\zeta+f)\right]
$$

In this study we calculated the RWS using monthly mean data. In general, the absolute vorticity is small in the tropics, which exhibits a poleward gradient. However, the divergent flow is largest around the edge of either side of the tropical heating maximum. Equation (4) describes that an equatorial region of tropical divergence situated even in ambient easterlies could produce a 
(a) <Q1>, PSI200, [T]500-200

JJA

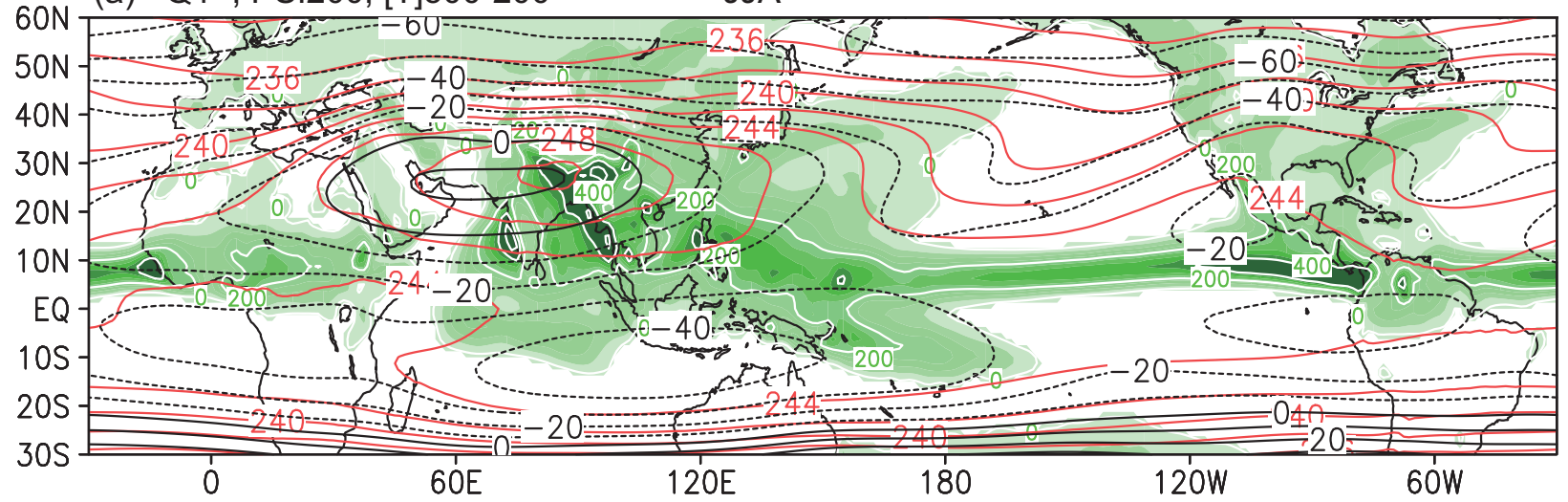

(b) <Q1>, PSI200, [T]500-200 DJF

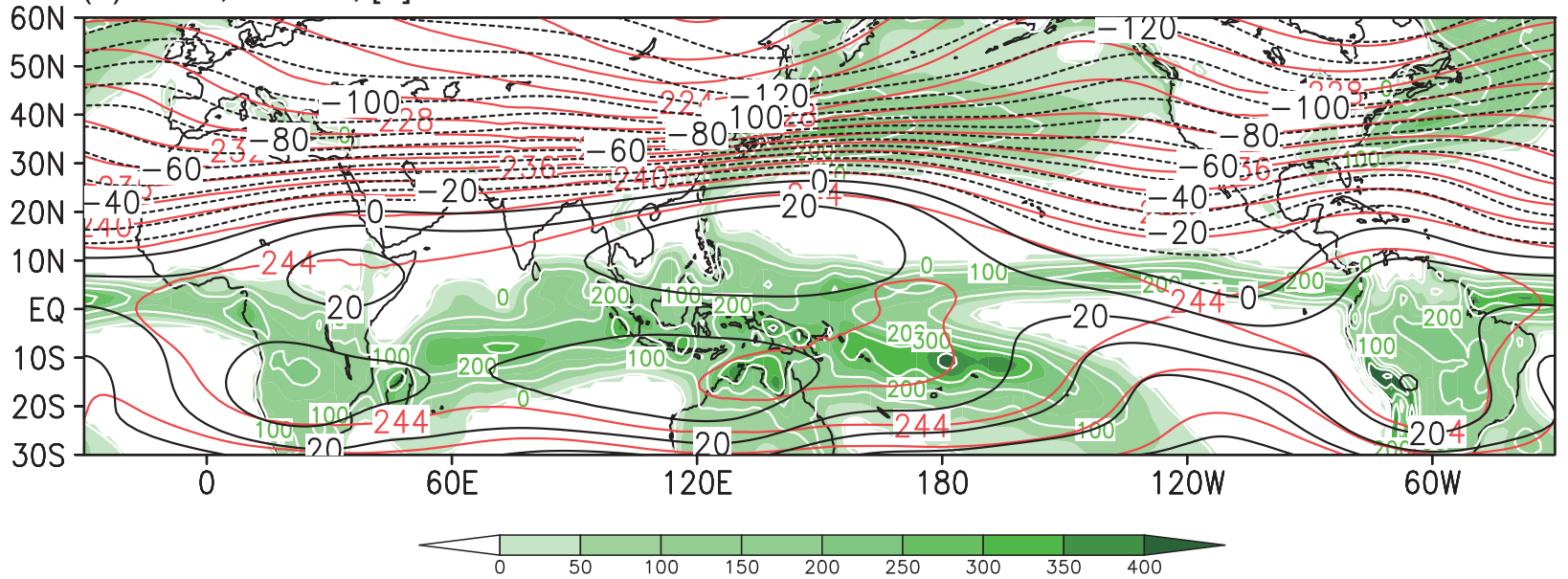

FIG. 2. Observational thermodynamic structure for (a) northern summer (June-August) and (b) southern summer (December-February). Black contours are the 200-hPa streamfunction $\left(1.0 \times 10^{6} \mathrm{~m}^{2} \mathrm{~s}^{-1}\right)$. Negative contours are indicated by dashed lines. Shading with white contours shows vertically integrated diabatic heating $\left\langle Q_{1}\right\rangle\left(\mathrm{W} \mathrm{m}^{-2}\right)$ calculated as a residual in the thermodynamic energy equation. Red contours are estimates of mass-weighed vertical mean temperature for 500-200 hPa (K).

large RWS in the subtropical westerlies due to the presence of large gradients in the background absolute vorticity.

The climatological RWS shows strong seasonality caused by the movement of heat sources that can be responsible for the various teleconnection patterns ( $\mathrm{Lu}$ and Kim 2004; Shimizu and de Albuquerque Cavalcati 2011). This perspective allows us to discuss the physical processes involved in the genesis of UTACs throughout the season.

\section{Structure of UTAC}

To discuss the thermodynamic structure of UTACs, the climatological 200-hPa streamfunction, the column heating $\left\langle Q_{1}\right\rangle$, and mass-weighted 500-200-hPa temperature for JJA and DJF are shown in Fig. 2. The most outstanding features for the JJA period are the large anticyclones centered over the southern edge of the Tibetan Plateau (black contour; corresponding to the $\mathrm{SAH}$ ), which coincides with the temperature maximum (red contour). As was mentioned in section 1, early studies have emphasized the thermal forcing of the Tibetan Plateau (e.g., Hahn and Manabe 1975). After the ECMWF reanalysis data became available, the summer monsoon rainfall located in a region extending from the Bay of Bengal to the southern Tibetan Plateau (green shading in Fig. 2a) has been considered an important factor for the formation of a warm core through the in situ condensation heating (Yanai et al. 1992). During the development of the summer monsoon from late April to early June, adiabatic descent, anchored with remote diabatic heating relevant to the Indo-China monsoon (Minoura et al. 2003), as well as the Hadley circulation (Tamura et al. 2010) contributes to the development of the warm center to some extent.

To identify the principal factors contributing to the heating or cooling during JJA, we visualized the vertical distributions of $Q_{1}$ together with the air temperature, geopotential height, and vertical wind averaged over $20^{\circ}-30^{\circ} \mathrm{N}$ (Fig. 3a) and $60^{\circ}-90^{\circ} \mathrm{E}$ (Fig. 4a). The warm core seen in Fig. 2a has a peak of approximately 250 $\mathrm{hPa}$ above the deep heating. The eastern part of the UTAC is characterized by deep heating throughout the troposphere 
(a) Z, T, Q1, Omega JJA

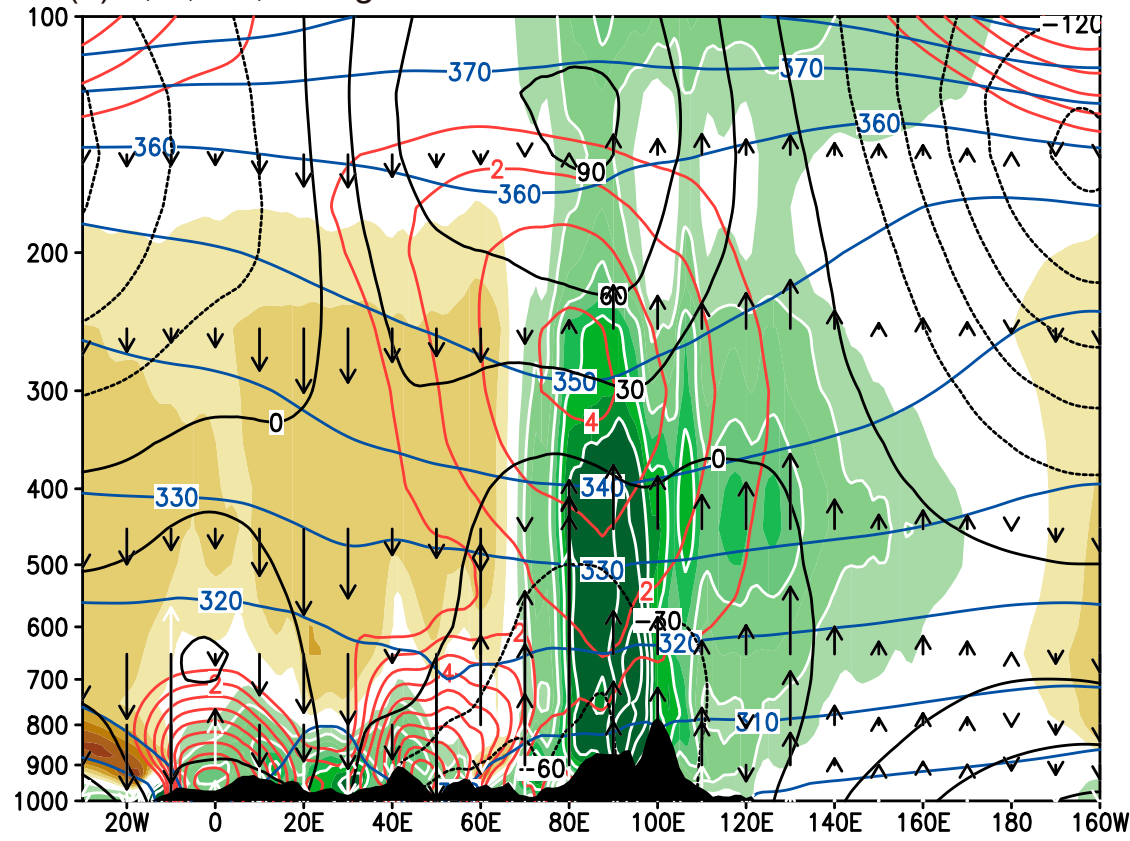

(b) Z, T, Q1, Omega DJF

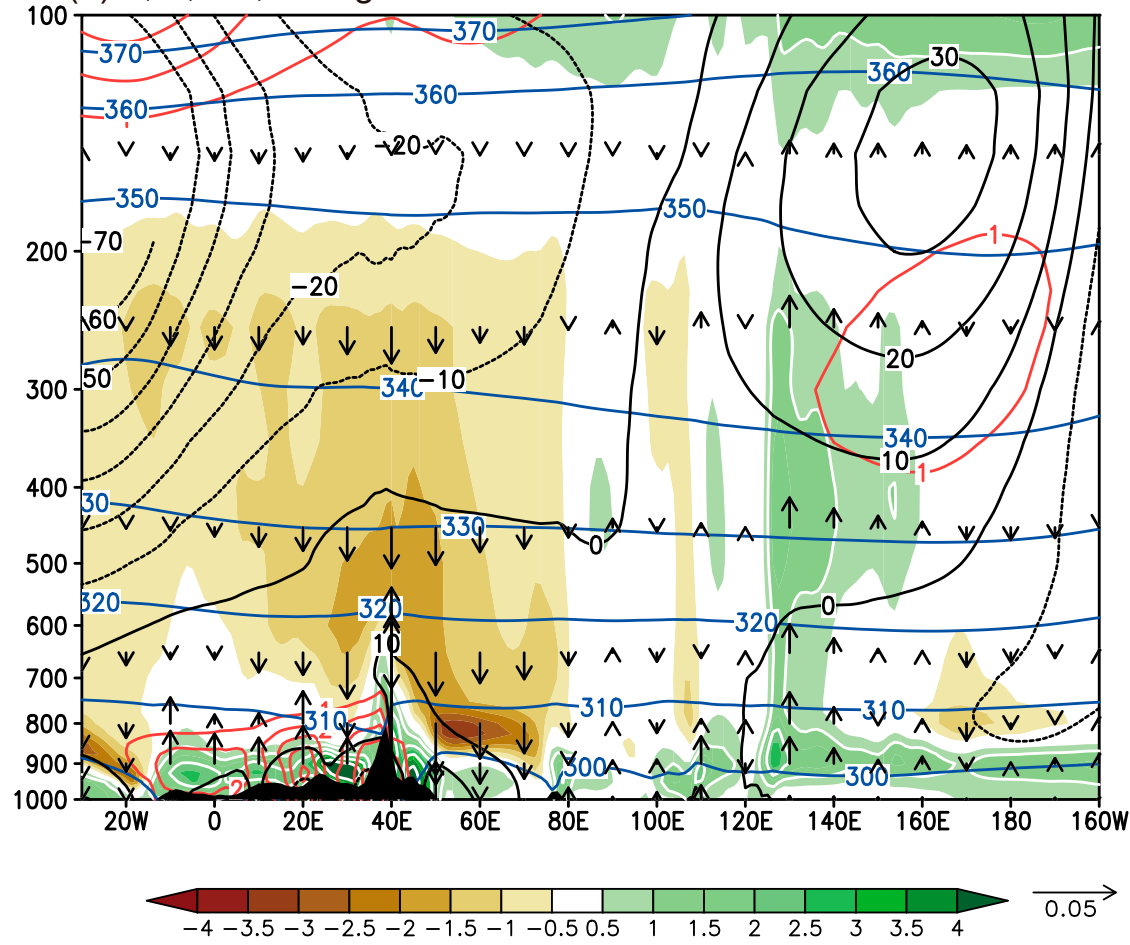

FIG. 3. Vertical structure of geopotential height (black contour; m), diabatic heating field (shading with white contour; $\mathrm{K}_{\text {day }}{ }^{-1}$ ), air temperature (red contour; $\mathrm{K}$ ) and potential temperature (blue contour; K) along (a) $20^{\circ}-30^{\circ} \mathrm{N}$ during June-August and (b) $5^{\circ}-15^{\circ} \mathrm{N}$ during December-February. The plotted geopotential height and temperature are deviations from the zonal mean. Vectors are vertical $p$ velocity (unit vector is $0.05 \mathrm{~Pa} \mathrm{~s}^{-1}$ ). The ordinate represents the vertical level (hPa) using a logarithmic scale. Data below the local surface are masked. 
(a) Z, T, Q1, Omega JJA 60-90E

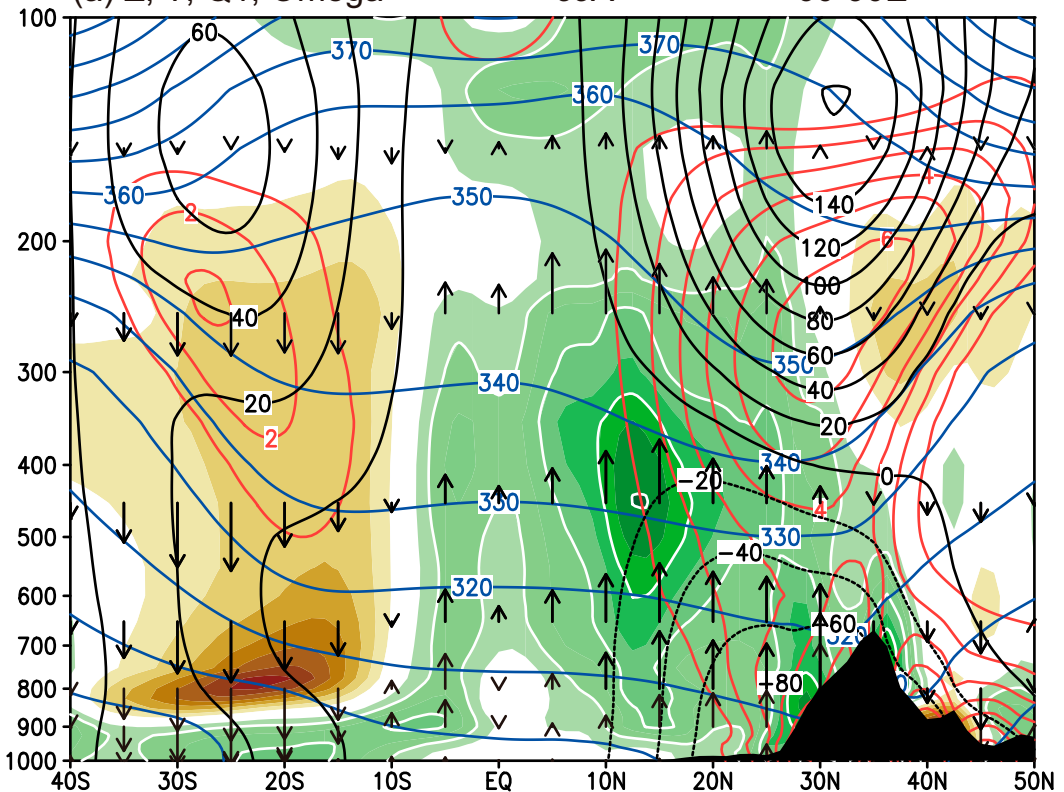

(b) Z, T, Q1, Omega

DJF

120-150E
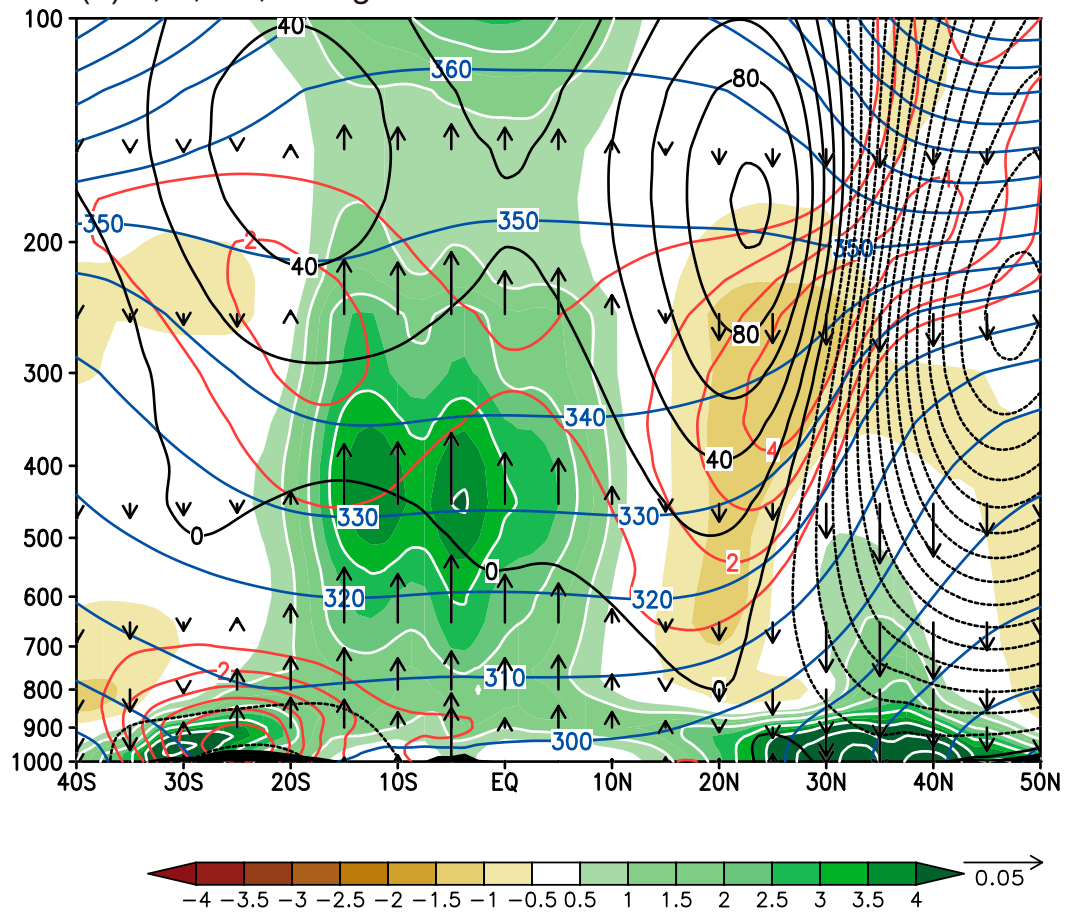

FIG. 4. Vertical structure of geopotential height (black contour; $\mathrm{m}$ ), diabatic heating field (shading with white contour; $\mathrm{K}$ day $^{-1}$ ), air temperature (red contour; $\mathrm{K}$ ), and potential temperature (blue contour; $\mathrm{K}$ ) along (a) $60^{\circ}-90^{\circ} \mathrm{E}$ during June-August and (b) $120^{\circ}-150^{\circ} \mathrm{E}$ during December-February. The plotted geopotential height and temperature are deviations from the zonal mean. Vectors are vertical $p$ velocity (unit vector is $0.05 \mathrm{~Pa} \mathrm{~s}^{-1}$ ). The plotted geopotential height and temperature are deviations from the zonal mean. The ordinate represents the vertical level $(\mathrm{hPa})$ using a logarithmic scale. Data below the local surface are masked. 
(a) RWS (JJA)

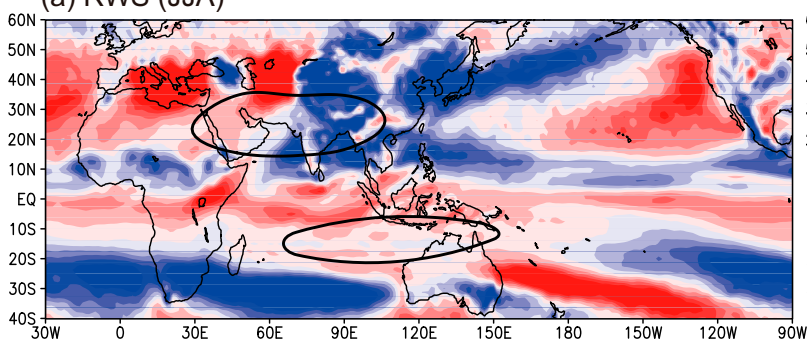

(c) Advection by divergent wind (JJA)
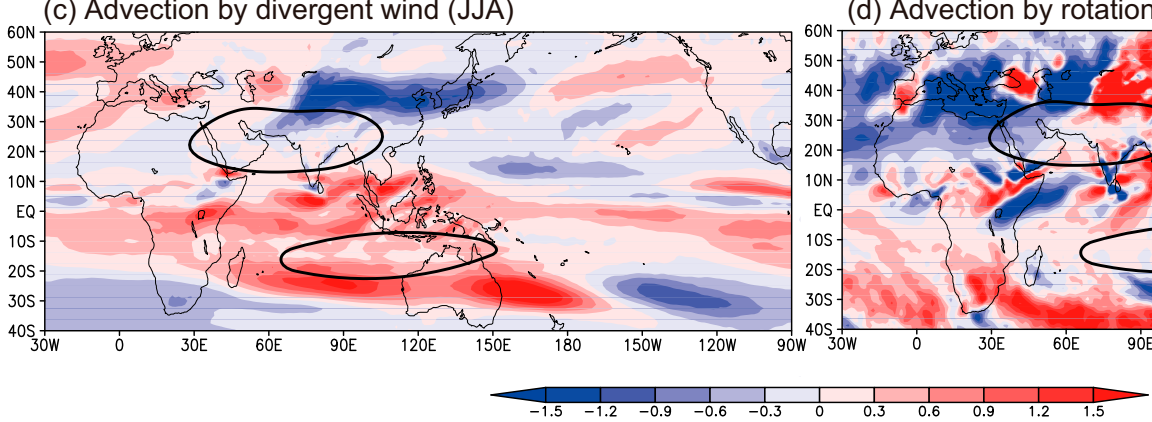

(e) Divergent wind (JJA)

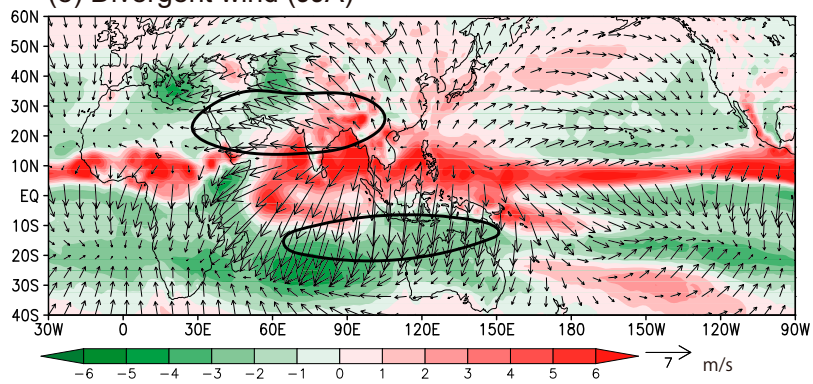

FIG. 5. The climatological distributions of 200-hPa vorticity and divergent fields of northern summer (June-August) UTAC. (a) The Rossby wave source, (b) the vortex stretching term, (c) the advection term by divergent wind, (d) the advection term by rotational wind, and (e) divergent wind and its divergence (shading; $10^{-6} \mathrm{~s}^{-1}$ ) computed from the velocity potential. The unit of vorticity tendency is $1.0 \times$ $10^{-10} \mathrm{~s}^{-2}$. The bold lines indicate the position of UTAC obtained from Fig. 1a (the streamfunction $=0$ in the $\mathrm{NH}$ and -40 in the SH).

centered between 400 and $500 \mathrm{hPa}$, accompanied by strong upward motion. This indicates that the upper-tropospheric temperature maxima are probably due to the release of latent heat from condensation caused by precipitation. Recently, Wu et al. (2015) demonstrated that the vertical gradient of monsoon diabatic heat induces a vertical wind shear (northerly in the upper troposphere and southerly in the lower troposphere) based on the Sverdrup balance. This vertical northerly wind shear is maintained by the thermal wind balance, which requires the eastward zonal temperature decrease, thus generating the upper-tropospheric temperature maxima to the west of the latent heating and the massive SAH aloft.

The other notable feature of Fig. 3a is the deepening of the isentropic surface (blue contour) under the UTAC, which is consistent with the warmth in the upper troposphere in view of hydrostatic balance. When this thermal structure interacts with the midlatitude subtropical jet, the air moves down to the sloped isentropic surface on the western side of the UTAC and backs up (b) Vortex streching (JJA)

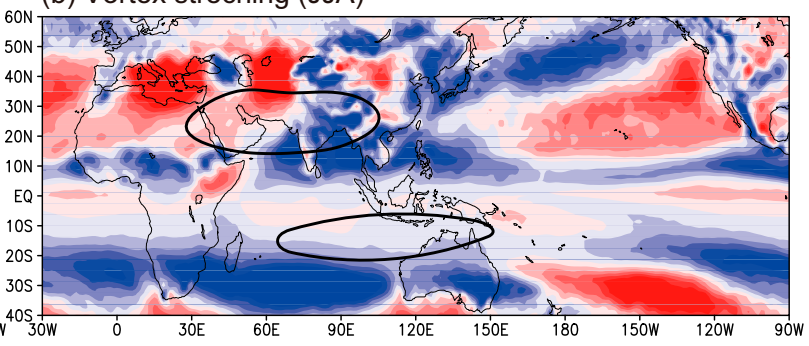

(d) Advection by rotational wind (JJA)

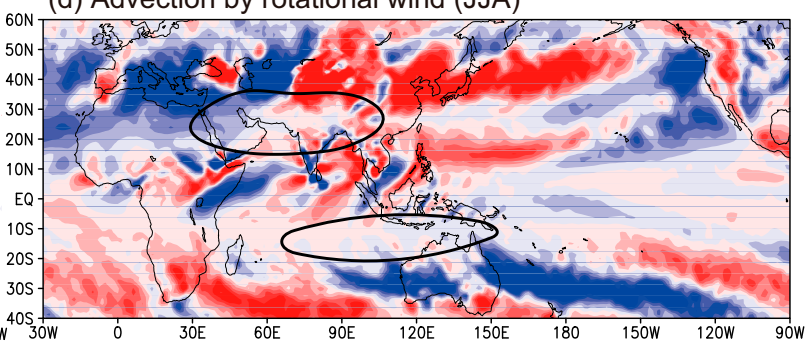


(a) RWS (DJF)

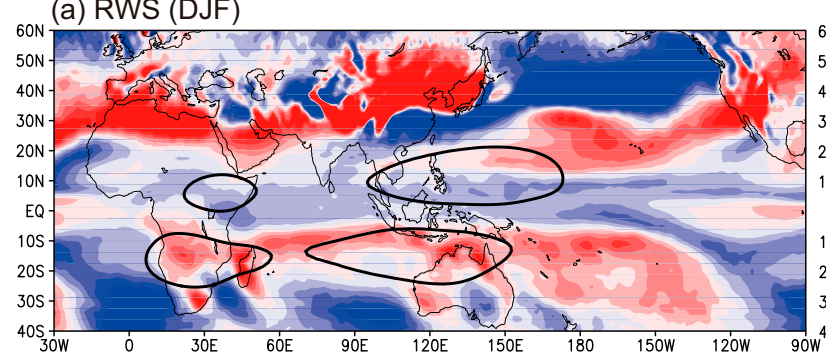

(c) Advection by divergent wind (DJF)

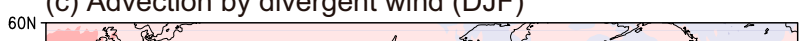

(b) Vortex streching (DJF)

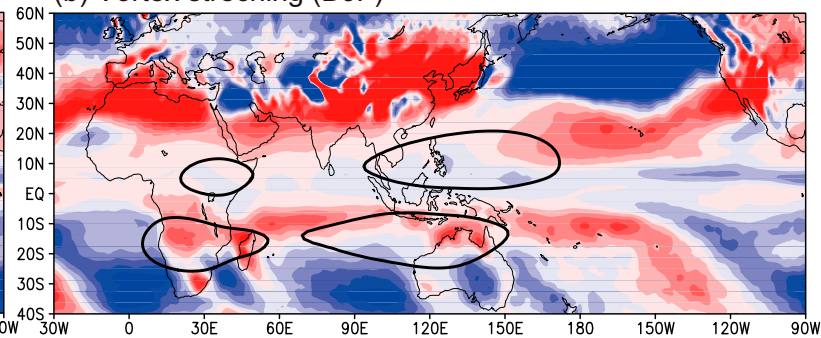

(d) Advection by rotational wind (DJF)

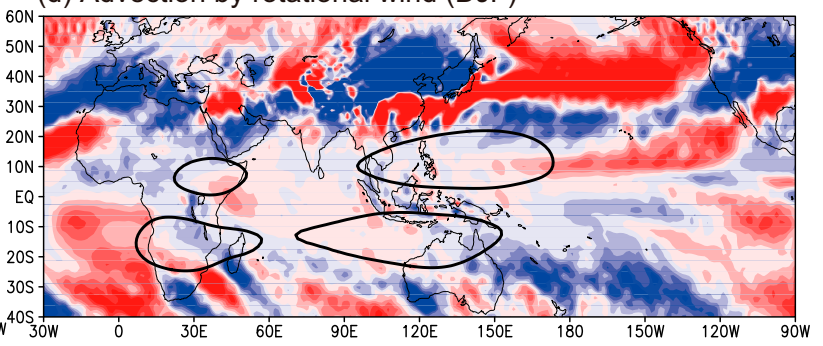

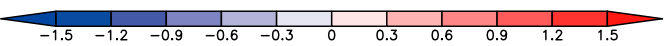

(e) Divergent wind (DJF)

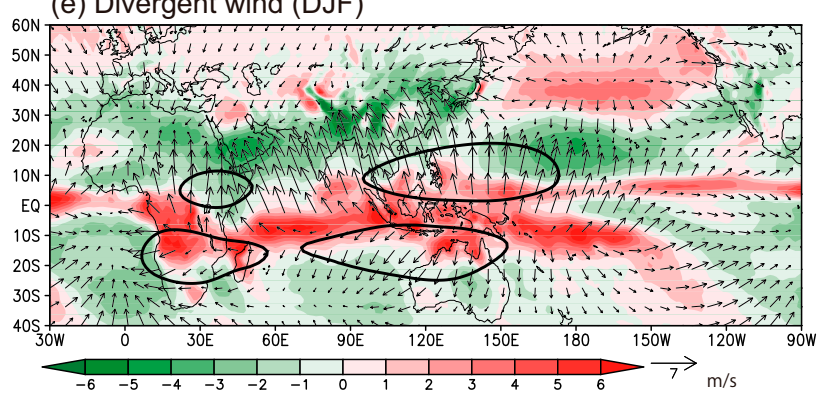

Fig. 6. As in Fig. 5, but for December-February. The bold lines indicate the position of UTAC obtained from Fig. 1b (the streamfunction $=20$ in the $\mathrm{NH}$ and 10 in the $\mathrm{SH}$ ).

Two UTACs reside on each side of the heating maxima $\left(\sim 5^{\circ} \mathrm{S}\right)$, mainly over the Maritime Continent and the western Pacific sector (Figs. 2b and 4b). The east-west asymmetric structure during JJA seen in Fig. 2a bears a resemblance to those in DJF (Fig. 3b), whereas the amplitude of diabatic heating accompanied by a rising motion and upper-tropospheric warmth are relatively small. In contrast, the paired rising $\left(20^{\circ} \mathrm{S}-10^{\circ} \mathrm{N}\right)$ and sinking $\left(>20^{\circ} \mathrm{N}\right)$ branches of the Hadley circulation during DJF (Fig. 4b) are reversed from those in JJA (upward over $10^{\circ}-25^{\circ} \mathrm{N}$ and descending over $>15^{\circ} \mathrm{S}$ ), which is anchored with enhanced convection in the SH (Fig. 4a). The downward motion occurs throughout the troposphere to the north of $10^{\circ} \mathrm{N}$ during DJF. Meanwhile, the temperature maxima are found over $20^{\circ}-40^{\circ} \mathrm{N}$ in the mid- to upper troposphere (Fig. 4b) with northward tilt, which is slightly different from those in the JJA configuration (Fig. 4a).

\section{Diagnosis of vorticity fields}

To better understand the causes of UTACs, we assessed the contributors to the local vorticity changes based on the Eqs. (3) and (4) for the northern summer (Fig. 5) and winter (Fig. 6), respectively. Looking at the RWS in JJA first (Fig. 5a), negative values extend over the Asian continent to the mid-Pacific. As shown in Figs. 5a and 5b, the patterns of vortex stretching around the northern summer UTAC are similar to those of RWS, except in the northeast of the UTAC. This indicates that the diabatic heating associated with monsoon precipitation is an important factor in the genesis of the northern summer UTAC. As for the SH over the Asian-Australian monsoon region, the southward advection of positive absolute vorticity (anticyclonic) by the divergent flow (Fig. 5c) appears to be the main contributor to the UTACs along $10^{\circ} \mathrm{S}$, as shown in Fig. 2a. Chen et al. (1989) noted that UTACs over the southern part of the African continent and South America have no Northern Hemisphere (NH) counterparts based on the WMONEX intensive observation period from December 1978 to January 1979. These obscure UTACs are consistent with relatively smaller southward advection of anticyclonic vorticity by the divergent winds. Chen et al. (1989) also showed that a pair of the UTACs over the Asian-western Pacific-Australian monsoon sectors accompany the low-level cyclonic circulation beneath, which can be interpreted as a Matsuno-Gill-type atmospheric response to the deep heating in the tropics. 

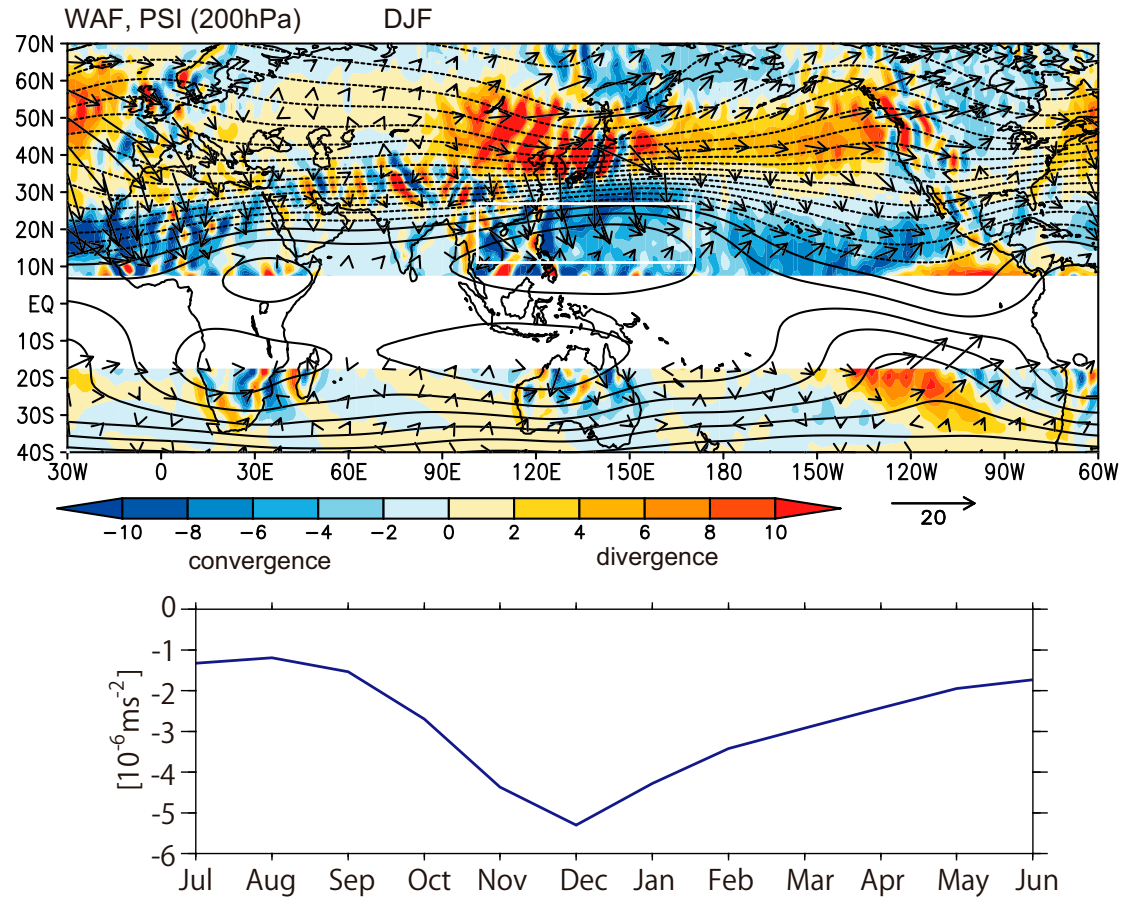

FIG. 7. Climatology of the wave activity flux $\mathbf{F}_{s}$ (vectors; $\mathrm{m}^{2} \mathrm{~s}^{-2}$ ) and its divergence (shading; $\left.1.0 \times 10^{-6} \mathrm{~m} \mathrm{~s}^{-2}\right)$. Contours are the streamfunction $\left(1.0 \times 10^{6} \mathrm{~m}^{2} \mathrm{~s}^{-1}\right)$ at $200 \mathrm{hPa}$ averaged over December-February. Latitudinal band of zonal mean zonal wind less than $5 \mathrm{~m} \mathrm{~s}^{-1}$ are masked. (bottom) The seasonal evolution of flux divergence $\left(1.0 \times 10^{-6} \mathrm{~m} \mathrm{~s}^{-2}\right)$ averaged over $\left(10^{\circ}-25^{\circ} \mathrm{N}\right.$, $100^{\circ}-170^{\circ} \mathrm{E}$ ) corresponding to the northern parts of the UTACs (white square).

Based on the map of the upper-tropospheric streamfunction (see Fig. 2b) in DJF reveals that the UTACs over the western North Pacific-especially its southern part-are consistent with negative RWS values (Fig. 6a). In contrast, the northern part of the winter hemisphere UTAC cannot be fully explained by negative vorticity anchored with the tropical convection and thereby requires alternative explanations, such as the simple Gill theory. As inferred from the advection term by the divergent wind (Fig. 6c), the negative values are prominent to the east of the UTAC around the date line $\left(150^{\circ} \mathrm{E}-120^{\circ} \mathrm{W}\right)$. At the same time, the negative vorticity is partially cancelled by the advection of positive vorticity by rotational winds (Fig. 6d), which could be associated with diminished UTACs. The DJF UTAC in the SH extending from the Indian Ocean to the western Pacific, including Australia (solid line in Fig. 6), is concurrent with the positive RWS in which vortex stretching is a major contributor. It should be mentioned here that positive RWS conditions also extend into the SH Pacific, which is offset by the advection of negative vorticity by rotational winds. Those features are consistent with the presence of enhanced convection relevant to the South Pacific convergence zone. In summary, the SAH and the SH summer UTAC accompany nearby heat sources, highlighting the important role of the Gill's dynamics. As for the SH UTAC during JJA, it can be understood by the interhemispheric advection of the anticyclonic vorticity anchored with the SAH. These results imply that the DJF UTAC in the NH and the
JJA UATC in the SH exhibit a dynamically unique feature among other UTACs over the Asian-western Pacific sector.

To understand the cause of the DJF UTAC in the NH, we examined the influence of higher latitudes associated with the East Asian winter monsoon. Several studies have demonstrated that planetary waves are excited from the cold land surface over the Eurasian continent and propagate upward, splitting into two branches in the upper troposphere, with one propagating into the stratosphere and the other into the tropics (e.g., Chen et al. 2005; Takaya and Nakamura 2013). One of the major analysis tools used to envisage the propagation of stationary eddies relative to the zonal mean flow is the threedimensional wave activity flux vector $\left(\mathbf{F}_{s}\right)$ on the log-pressure coordinate proposed by Plumb (1985), which is defined as

$$
\mathbf{F}_{S}=p^{*} \cos \phi\left\{\begin{array}{c}
v^{2}-\frac{1}{2 \Omega a \sin 2 \phi} \frac{\partial\left(v^{\prime} \Phi^{\prime}\right)}{\partial \lambda} \\
-u^{\prime} v^{\prime}+\frac{1}{2 \Omega a \sin 2 \phi} \frac{\partial\left(u^{\prime} \Phi^{\prime}\right)}{\partial \lambda} \\
\frac{2 \Omega a \sin \phi}{S}\left[v^{\prime} T^{\prime}-\frac{1}{2 \Omega a \sin 2 \phi} \frac{\partial}{\partial \lambda}\left(T^{\prime} \Phi^{\prime}\right)\right]
\end{array}\right\}
$$

where $\Omega$ is the angular speed of rotation of Earth, $a$ is the radius of Earth, $\Phi$ is geopotential, $\phi$ is latitude, $\lambda$ is longitude, and $p^{*}$ is pressure normalized by $1000(\mathrm{hPa})$. The primes 


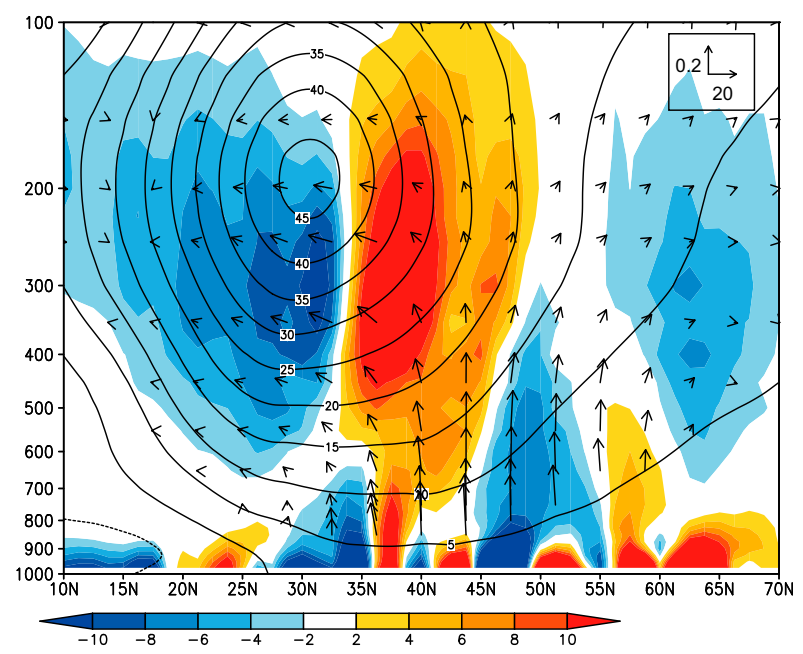

FIG. 8. Climatology of latitude-height projections of $\mathbf{F}_{s}$ (vectors; $\mathrm{m}^{2} \mathrm{~s}^{-2}$ ) and its horizontal divergence (every $2.0 \times 10^{-6} \mathrm{~m} \mathrm{~s}^{-2}$, with positive values in red and negative values in blue) along $120^{\circ}-150^{\circ} \mathrm{E}$ averaged over December-February. Black contours denote the climatological zonal winds. The ordinate represents the vertical level $(\mathrm{hPa})$ using a logarithmic scale.

denote deviations from zonal mean values. The term $S$ is the static stability defined as

$$
S=\frac{\partial \hat{T}}{\partial z}+\frac{\kappa \hat{T}}{H},
$$

where a caret $\left(\%\right.$ signifies the regional average north of $20^{\circ} \mathrm{N}, H$ is a constant-scale height of $8000 \mathrm{~m}$, and the longitudinal integration of $\mathbf{F}_{s}$ corresponds to the Eliassen-Palm (EP) flux in spherical quasigeostrophic form [Eqs. (5) and (6)].

The two-dimensional horizontal wave activity flux (Plumb $1985)$ at $200 \mathrm{hPa}$ in DJF is illustrated in Fig. 7 based on the monthly mean field. Throughout the northern winter, an eastward flux is evident along the $40^{\circ}-50^{\circ} \mathrm{N}$ accompanying the wave trains. The zonally oriented wave train can be attributed to the waveguide effect of background westerlies. It is interesting to note that the planetary wave emanates from the cold Eurasian continent, which diverts southward approximately to the east of $120^{\circ} \mathrm{E}$, corresponding to the northern part of the UTAC. These results are more clearly shown in the meridional cross sections of $\mathbf{F}_{s}$ averaged over the longitude bands of $100^{\circ}$ and $150^{\circ} \mathrm{E}$ (Fig. 8). A significant portion of the upward wave activity flux can be seen over the midlatitude around $50^{\circ} \mathrm{N}$. The wave activity flux in the extratropical troposphere above the $500 \mathrm{hPa}$ turns toward the tropics, and converging at approximately $30^{\circ} \mathrm{N}$ and $300 \mathrm{hPa}$, which in turn decelerates the westerlies. Thus, the locations of the convergence around $20^{\circ} \mathrm{N}$ and $250 \mathrm{hPa}$ appear to be responsible for the decrease in the subtropical jet, which can account for the formation of the northern part of the UTACs. The seasonal evolution of the flux divergence over the northern part of the UTACs (the bottom panel in Fig. 7) clearly shows that enhancement of convergence takes place in the $\mathrm{NH}$ winter with peak in December, which is consistent with the emergence of the $\mathrm{NH}$ winter UTAC.

\section{Conclusions}

We investigated the geographical structure of UTACs throughout the season in the globe based on observational data, then revealed the inherent physical causality with the particular focus on the three types of UTACs over the Asian-Australian-western Pacific sector, which are schematically shown in Fig. 9. The northern summer UTAC over Asia, traditionally referred to as the Tibetan high or SAH, is caused by a heat-induced atmospheric Rossby response associated with Asian summer monsoon rainfall. The vigorous northern summer UTACs accompany upper-tropospheric temperature maxima with vertical northerly wind shear maintained by the thermal wind balance. The warmth of UTACs deepens the isentropic surface, which facilitates subsidence along its slope on the western side of the UTACs. Overall, the geographical configuration of wet and dry climates is consistent with recent progress in the understanding of UTACs (e.g., Rodwell and Hoskins 1996; Wu et al. 2015). Based on the diagnosis of vorticity fields, the summertime NH UTACs can be explained by the RWS, especially the stretching term. Meanwhile, we also show that anticyclonic vorticity in the upper troposphere caused by the deep heating of the Asian and western Pacific monsoon is transported by interhemispheric divergent winds toward the SH (e.g., Trenberth et al. 2000), thereby creating the SH UTACs.

As for the genesis of the northern winter UTACs over the subtropical western North Pacific, the negative RWS values along $0^{\circ}-10^{\circ} \mathrm{N}$ (Fig. 6a) account for the southern parts of the UTACs, in which the northward advection of anticyclonic vorticity anchored with the tropical convection is an important factor alongside vortex stretching. The interhemispheric divergent wind corresponds to part of the Hadley circulation. The absence of a negative RWS in the northern part of the UTACs requires a mechanism other than the meridional advection of absolute vorticity originating from the tropics. The analysis of the wave activity flux based on Plumb (1985) indicates that the equatorward propagation of the planetary waves emitted from the cold Eurasian continent and its convergence associated with decreased westerlies is a potentially important factor for the maintenance of the northern parts of the UTACs. Thus, the wintertime anticyclones emerging over the subtropical western North Pacific are caused by the remote influence anchored with the tropical convection and the cold Eurasian continent in which atmospheric teleconnections are important. These configurations indicate that the formation mechanisms of the winter UTACs are different from those of SAH and SH winter UTACs.

It has been widely accepted that surface subtropical anticyclones correspond to the descending branch of the Hadley cell, while UTACs cannot be directly explained by this meridional circulation. The NH winter (DJF) UTACs as well as SH winter (JJA) UTACs do not accompany nearby heat sources like the SAH. In contrast, the SH summer (DJF) UTACs emerge close to the diabatic heating, which is consistent with RWS patterns. Thus, our results imply that the winter UTACs are likely the result of not only the "Matsuno-Hoskins-Gill" picture (Matsuno 1966; Hoskins et al. 1977; Gill 1980) but also the influence of higher latitudes. 

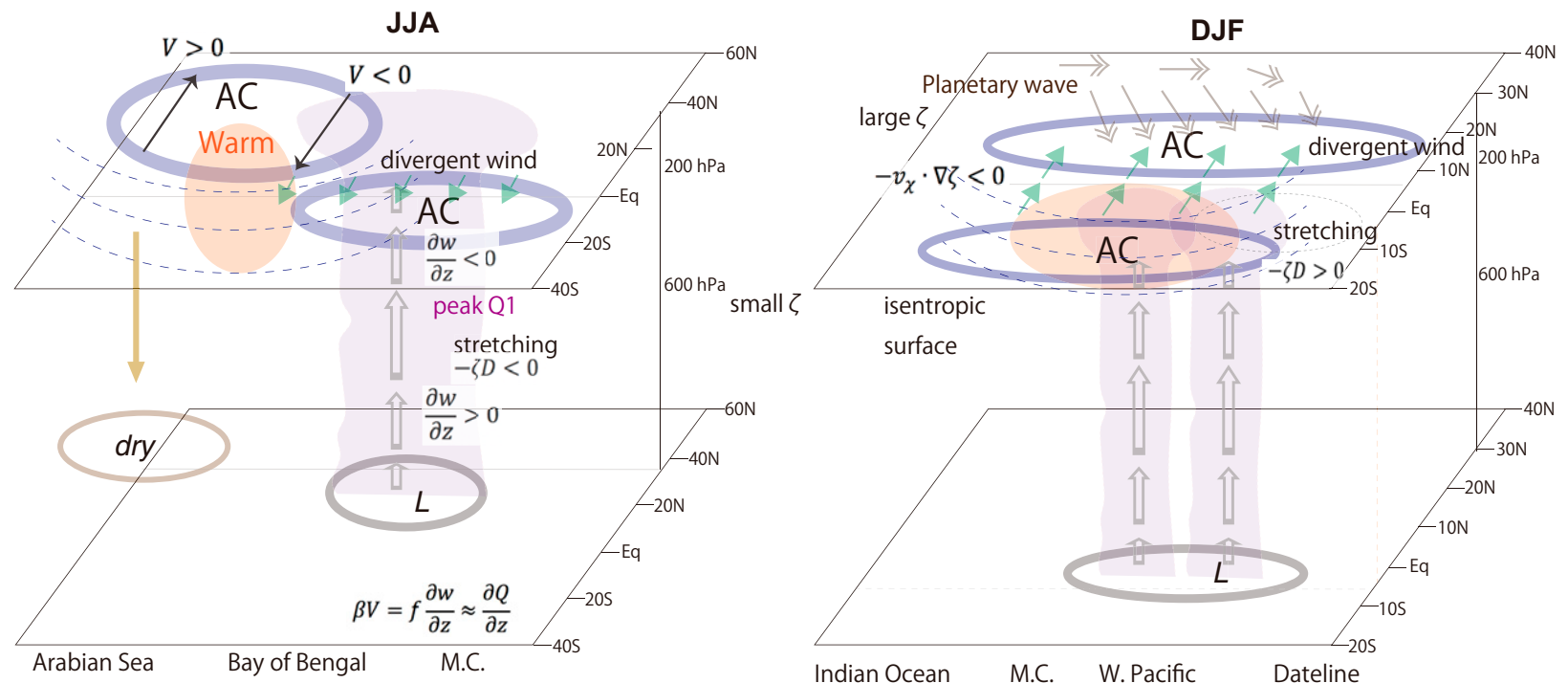

FIG. 9. Schematic illustration of the generation of UTACs over the Asian-western Pacific sector for (a) June-August and (b) December-February. Reddish shading shows cumulus convection. Dashed contours are isentropic surfaces.

In this study, we have proposed a viewpoint of the climatological planetary wave for interpreting the establishment and maintenance of the subtropical UTAC. Holton and Colton (1972) examined the maintenance of the Tibetan high with linearized vorticity budget analysis, suggesting that cumulous frictional dissipation should be balanced with vorticity generation including vortex stretching and vorticity advection. Similarly, the question remains as to whether wintertime UTACs are indeed the manifestation of high-frequency transient eddies (Plumb 1986; Trenberth 1986), because no clarification of their causality has yet been made.

Previous studies (Chen et al. 1999; Chen 2003) have shown that the summer hemisphere is characterized by a spatially quadrature relationship through the east-west circulation. Our results highlight the combination of the meridional advection of anticyclonic vorticity by the divergent winds from the tropics and the propagation of planetary waves from higher latitudes. Based on this, future studies focusing on tropicalextratropical interactions are encouraged, both in the contexts of classical climatology and over a range of temporal scales.

Acknowledgments. This work was supported by the Japan Society for the Promotion of Science (JSPS) Grants-in-Aid for Scientific Research (KAKENHI), Grant 7K01223/ $21 \mathrm{H} 00626$, and by the industry-academia collaboration project for the anomalous warm winter 2019/2020 of University of Tsukuba. We would also like to thank Editage for editing and reviewing this manuscript for English language.

\section{REFERENCES}

Abe, M., A. Kitoh, and T. Yasunari, 2003: An evolution of the Asian summer monsoon associated with mountain uplift-Simulation with the MRI atmosphere-ocean coupled GCM. J. Meteor. Soc. Japan, 81, 909-933, https://doi.org/10.2151/jmsj.81.909.
Boos, W. R., and Z. Kuang, 2013: Sensitivity of South Asian monsoon to elevated and non-elevated heating. Sci. Rep., 3, 1192, https://doi.org/10.1038/srep01192.

Chen, T.-C., 2003: Maintenance of summer monsoon circulations: A planetary perspective. J. Climate, 16, 2022-2037, https://doi. org/10.1175/1520-0442(2003)016<2022:MOSMCA > 2.0.CO;2.

— M. C. Yen, and H. van Loon, 1989: The effect of the divergent circulation on some aspects of the 1978/79 Southern Hemisphere monsoon. J. Climate, 2, 1270-1288, https://doi. org/10.1175/1520-0442(1989)002<1270:TEOTDC >2.0.CO;2.

_ - S. P. Weng, and S. Schubert, 1999: Maintenance of austral summertime upper-tropospheric circulation over tropical South America: Bolivian high-Nordeste low system. J. Atmos. Sci., 56, 2081-2100, https://doi.org/10.1175/1520-0469(1999)056 $<2081$ :MOASUT $>2.0$.CO;2.

Chen, W., S. Yang, and R.-H. Huang, 2005: Relationship between stationary planetary wave activity and the East Asian winter monsoon. J. Geophys. Res., 110, D14110, https://doi.org/10. 1029/2004JD005669.

Flohn, H., 1957: Large-scale aspects of the summer monsoon in South and East Asia. J. Meteor. Soc. Japan, 35A, 180-186, https://doi.org/10.2151/jmsj1923.35A.0_180.

Gill, A. E., 1980: Some simple solutions for heat-induced tropical circulation. Quart. J. Roy. Meteor. Soc., 106, 447-462, https:// doi.org/10.1002/qj.49710644905.

Hahn, D. G., and S. Manabe, 1975: The role of mountains in South Asian monsoon circulation. J. Atmos. Sci., 32, 1515-1541, https:// doi.org/10.1175/1520-0469(1975)032<1515:TROMIT>2.0.CO;2.

Holton, J. R., and D. E. Colton, 1972: A diagnostic study of the vorticity balance at $200 \mathrm{mb}$ in the tropics during northern summer. J. Atmos. Sci., 29, 1124-1128, https://doi.org/10.1175/ 1520-0469(1972)029<1124:ADSOTV>2.0.CO;2.

Hoskins, B., and D. J. Karoly, 1981: The steady linear response of a spherical atmosphere to thermal and orographic forcing. $J$. Atmos. Sci., 38, 1179-1196, https://doi.org/10.1175/1520-0469 (1981)038<1179:TSLROA > 2.0.CO;2. , and M. J. Rodwell, 1995: A model of the Asian summer monsoon. Part I: The global scale. J. Atmos. Sci., 52, 1329-1340, 
https://doi.org/10.1175/1520-0469(1995)052<1329:AMOTAS > 2. $0 . \mathrm{CO} ; 2$.

—, A. Simmons, and D. Andrews, 1977: Energy dispersion in a barotropic atmosphere. Quart. J. Roy. Meteor. Soc., 103, 553-567, https://doi.org/10.1002/qj.49710343802.

Kobayashi, S., and Coauthors, 2015: The JRA-55 reanalysis: General specifications and basic characteristics. J. Meteor. Soc. Japan, 93, 5-48, https://doi.org/10.2151/jmsj.2015-001.

Krishnamurti, T. N., 1971a: Observational study of the tropical upper tropospheric motion during the Northern Hemisphere summer. J. Appl. Meteor., 10, 1066-1096, https://doi.org/10. 1175/1520-0450(1971)010<1066:OSOTTU>2.0.CO;2.

— 1971b: Tropical east-west circulation during the northern summer. J. Atmos. Sci., 28, 1342-1347, https://doi.org/10.1175/ 1520-0469(1971)028<1342:TEWCDT>2.0.CO;2.

- 1985: Summer monsoon experiment-A review. Mon. Wea. Rev., 113, 1590-1626, https://doi.org/10.1175/15200493(1985)113<1590:SMER > 2.0.CO;2.

Lau, K. M., and P. H. Chan, 1983: Short-term climate variability and atmospheric teleconnections from satellite-observed outgoing longwave radiation. Part I: Simultaneous relationships. J. Atmos. Sci., 40, 2751-2767, https://doi.org/10.1175/15200469(1983)040<2751:STCVAA > 2.0.CO;2.

Liebmann, B., and C. A. Smith, 1996: Description of a complete (interpolated) outgoing longwave radiation dataset. Bull. Amer. Meteor. Soc., 77, 1275-1277.

Lu, R., and B.-J. Kim, 2004: The climatological Rossby wave source over the STCZs in the summer Northern Hemisphere. J. Meteor. Soc. Japan, 82, 657-669, https://doi.org/10.2151/ jmsj.2004.657.

Matsuno, T., 1966: Quasi-geostrophic motions in the equatorial area. J. Meteor. Soc. Japan, 44, 25-43, https://doi.org/10.2151/ jmsj1965.44.1_25.

Minoura, D., R. Kawamura, and T. Matsuura, 2003: A mechanism of the onset of South Asian summer monsoon. J. Meteor. Soc. Japan, 81, 563-580, https://doi.org/10.2151/jmsj.81.563.

Murakami, T., 1987: Effects of the Tibetan Plateau. Monsoon Meteorology, C. P. Chang and T. Krishnamurti, Eds., Oxford University Press, 235-270.

Ose, T., 1998: Seasonal change of Asian summer monsoon circulation and its heat source. J. Meteor. Soc. Japan, 76, 1045-1063, https://doi.org/10.2151/jmsj1965.76.6_1045.

Plumb, R. A., 1985: On the three-dimensional propagation of stationary waves. J. Atmos. Sci., 42, 217-229, https://doi.org/10. 1175/1520-0469(1985)042<0217:OTTDPO > 2.0.CO;2.

— 1986: Three-dimensional propagation of transient quasi-geostrophic eddies and its relationship with the eddy forcing of the time-mean flow. J. Atmos. Sci., 43, 1657-1678, https://doi. org/10.1175/1520-0469(1986)043<1657:TDPOTQ>2.0.CO;2.

Rodwell, M., and B. Hoskins, 1996: Monsoons and the dynamics of deserts. Quart. J. Roy. Meteor. Soc., 122, 1385-1404, https://doi.org/10.1002/qj.49712253408.

Sardeshmukh, P. D., and B. J. Hoskins, 1985: Vorticity balances in the tropics during the 1982-83 El Niño-Southern Oscillation event. Quart. J. Roy. Meteor. Soc., 111, 261-278, https:// doi.org/10.1256/smsqj.46801.

- and — , 1988: The generation of global rotational flow by steady idealized tropical divergence. J. Atmos. Sci., 45, 1228-
1251, https://doi.org/10.1175/1520-0469(1988)045<1228:TGOG $\mathrm{RF}>2.0 . \mathrm{CO} ; 2$.

Shimizu, H. M., and I. F. de Albuquerque Cavalcati, 2011: Variability patterns of Rossby wave source. Climate Dyn., 37, 441-454, https://doi.org/10.1007/s00382-010-0841-z.

Takaya, K., and H. Nakamura, 2013: Interannual variability of the East Asian winter monsoon and related modulations of the planetary waves. J. Climate, 26, 9445-9461, https://doi.org/10. 1175/JCLI-D-12-00842.1.

Tamura, T., K. Taniguchi, and T. Koike, 2010: Mechanism of upper tropospheric warming around the Tibetan Plateau at the onset phase of the Asian summer monsoon. J. Geophys. Res., 115, D02106, https://doi.org/10.1029/ 2008JD011678.

Trenberth, K. E., 1986: An assessment of the impact of transient eddies on the zonal flow during a blocking episode using localized Eliassen-Palm flux diagnostics. J. Atmos. Sci., 43, 2070-2087, https://doi.org/10.1175/1520-0469(1986)043<2070: AAOTIO $>2.0 . \mathrm{CO} ; 2$.

—, G. W. Branstator, D. Karoly, A. Kumar, N.-C. Lau, and C. Ropelewski, 1998: Progress during TOGA in understanding and modeling global teleconnections associated with tropical sea surface temperatures. J. Geophys. Res., 103, 1429114324, https://doi.org/10.1029/97JC01444.

—, D. P. Stepaniak, and J. M. Caron, 2000: The global monsoon as seen through the divergent atmospheric circulation. J. Climate, 13, 3969-3993, https://doi.org/10.1175/1520-0442 (2000)013<3969:TGMAST>2.0.CO;2.

Ueda, H., H. Kamahori, and N. Yamazaki, 2003: Seasonal contrasting features of heat and moisture budgets between the eastern and western Tibetan Plateau during the GAME IOP. J. Climate, 16, 2309-2324, https://doi.org/10.1175/2757.1.

Wang, B., J. Liu, H.-J. Kim, P. J. Webster, and S. Y. Yim, 2012: Recent change of the global monsoon precipitation (1979-2008). Climate Dyn., 39, 1123-1135, https://doi.org/ 10.1007/s00382-011-1266-z.

Wu, G., and Y. Liu, 2003: Summertime quadruplet heating pattern in the subtropics and the associated atmospheric circulation. Geophys. Res. Lett., 30, 1201, https://doi.org/10.1029/ 2002GL016209.

— , B. He, Y. Liu, Q. Bao, and R. Ren, 2015: Location and variation of the summertime upper-troposphere temperature maximum over South Asia. Climate Dyn., 45, 2757-2774, https://doi.org/10.1007/s00382-015-2506-4.

Yanai, M., and T. Tomita, 1998: Seasonal and interannual variability of atmospheric heat sources and moisture sinks as determined from NCEP-NCAR reanalysis. J. Climate, 11, 463-482, https://doi.org/10.1175/1520-0442(1998)011<0463: SAIVOA $>2.0 . \mathrm{CO} ; 2$.

—, S. Esbensen, and J.-H. Chu, 1973: Determination of bulk properties of tropical cloud clusters from large-scale heat and moisture budgets. J. Atmos. Sci., 30, 611-627, https://doi.org/ 10.1175/1520-0469(1973)030<0611:DOBPOT>2.0.CO;2.

— C. F. Li, and Z. S. Song, 1992: Seasonal heating of the Tibetan Plateau and its effects on the evolution of the Asian summer monsoon. J. Meteor. Soc. Japan, 70, 319-351, https:// doi.org/10.2151/jmsj1965.70.1B_319. 